\title{
Production of Biochar Based Porous Carbon Nanofibers for High-Performance Supercapacitor Applications
}

\author{
Shuangning Xiu ${ }^{1, a}$, Spero Gbewonyob ${ }^{2, a}$, Abolghasem Shahbazi ${ }^{1, *}$, Lifeng Zhang ${ }^{2, *}$
}

1. Biological Engineering Program, Department of Natural Resource and Environmental Design, North Carolina A\&T State University, Greensboro, NC, U.S.A

2. Joint School of Nanoscience and Nanotechnology, North Carolina A\&T State University, Greensboro, NC, U.S.A

${ }^{a}$ These two authors contributed equally to this work.

Received February 10, 2019; Accepted April 4, 2019; Published April 8, 2019

\begin{abstract}
Biomass-derived biochar was used as the precursor to synthesize porous carbons for supercapacitor electrodes. The biochar was first activated with $\mathrm{KOH}$ to generate porous carbon material and then fabricated into highly flexible porous carbon nanofibers (ECNF) by electrospinning technique. Activated carbons with a surface area of around $2258 \mathrm{~m}^{2} / \mathrm{g}$ were found. The resultant biochar based ECNF mats exhibited outstanding mechanical flexibility and electrochemical properties as free-stranding and binder free electrodes of supercapacitor. The PAN/BCK3 ECNFs, which were made from the composite of polyacrylonitrile (PAN) and $\mathrm{KOH}$-activated biochar (mass ratio of Biochar $/ \mathrm{KOH}=1: 3$ ) exhibited the highest gravimetric capacitance (108 $\mathrm{F} / \mathrm{g}$ at current density of $0.5 \mathrm{~A} / \mathrm{g}$ ) with high retention $(96 \%$ at $1 \mathrm{~A} / \mathrm{g})$ due to its well-developed micro-mesoporosity. The results indicated that biomass-derived biochar is a promising material which can be used for the production of low cost high performance electrode materials for supercapacitor.
\end{abstract}

Keywords: Biochar; Pyrolysis; Supercapacitor; Nanofiber; Activated carbon

\section{Introduction}

The excessive burning of fossil fuel has caused serious problems such as the global warming, environmental pollution, and energy crisis. Sustainable development requires discovering renewable and environmentally friendly technologies with the aim of resolving these problems. Among various newly developed energy technologies, supercapacitor, an energy storage device, has received great attention due to its highpower density, long cycle life, and quick charge/discharge capability [1, 2]. Supercapacitor can be used as uninterruptible power sources in electric vehicles, digital communications system, pulsing techniques, etc. [3, 4]. The microstructure of supercapacitor electrodes has a great influence on supercapacitor performance. Carbon material with high surface area and rich porous structure are the primary raw materials for making supercapacitors due to its high electric conductivity, strong mechanical property, wide availability, and low environmental impacts [5, 6]. However, high quality carbon materials like fullerene, graphene, and carbon nanotubes are hard to synthesize 
and expensive for massive production [6,7]. Therefore, producing attractive, high quality carbon materials from sustainable and low cost precursors is critical for the development of the supercapacitor industry [8].

Recently, biochar, a product from biomass thermochemical conversion, has received increasing attention for the use in several applications due to the cheap, abundant, and sustainable advantages [9]. In general, the bio-char is considered as waste byproduct or low value product [10]. The most common biochar application is soil amendment to mitigate greenhouse gas emission and improve soil health. Recent developments in activation procedures and/or precursors allow a better control over the pore structure and surface property. These characteristics have widened the use of biochar to more demanding applications, including using biochar as a precursor for making catalysts, energy storage, gas storage and contaminant adsorbents [9]. These new high-value applications are still in their infancy, and further research and development are needed to reach commercialization.

Up to now, various type porous carbon based electrode materials have been reported in the literature, such as spherical carbon [11], fibrous carbon [12], powdery carbon [13] et al. Among which, activated carbon fibers attract increasing interests owning to their unique microspores, light weight, free standing, high strength, fatigue endurance, and thermal resistance [14]. However, binders (organic/polymeric materials) are typically required for binding the powders/particles of the carbons to prepare the freestanding electrodes [15]. Such binders would reduce the overall performance of the electrodes. Hence, it is still a technological challenge to develop mechanically flexible electrodes with superior electrochemical performance.

The recent reported freestanding electrospun carbon nanofibers (ECNFs) prepared by electrospinning technique showed some promising advantages in the preparation process, ion transmission, and cost for using as free-standing, and binder-free electrode materials $[12,16]$. However, the specific surface area (SSA) of ECNF $\left(10-30 \mathrm{~m}^{2} / \mathrm{g}\right)$ is much lower than those of graphene $\left(272 \mathrm{~m}^{2} / \mathrm{g}\right)$ and carbon nanotubes $\left(909 \mathrm{~m}^{2} / \mathrm{g}\right)[16,17]$. To improve the specific capacitance and energy density of ECNFs based supercapacitors, many researchers have tried to incorporate some conductive and flexible materials such as graphene nanosheet, cobalt nitrite, and transition metal oxidizes to improve the SSA, flexibility, and conductivity of ECNF [16, 18, 19]. Although the performance of ECNF materials has been greatly improved, it still remains a challenge to prepare a flexible and low cost ECNF for energy storage systems due to the rigid nature of the metal materials, poor electrical conductivity, high cost, and toxicity.

In this work, biomass-derived biochar was used as the precursor to synthesize porous carbons for supercapacitor electrodes. Highly flexible porous biochar based ECNFs were for the first time fabricated by electrospinning technique and explored as electrode material. The main advantage of the prepared carbon material is its source (i.e., abundant/inexpensive biochar), and the biochar-based ECNFs can be directly used as binder-free electrode for high performance supercapacitor electrodes.

\section{Materials and Methods}

\subsection{Biochar and Activation}


The raw biochar was prepared by fast pyrolysis of loblolly pine sawdust, which was followed by sieving into the particle size range of $0.18-0.25 \mathrm{~mm}$ [20]. The biochar based actived carbons were prepared from raw biochar via $\mathrm{KOH}$ activation method. Typically, the raw biochar was mixed with potassium hydroxide $(\mathrm{KOH}$, Fisher Scientific Inc.) in a different mass ratio $(1: 1,1: 2,1: 3)$ and small amount of water in a crucible. Then the crucible was placed in a muffle furnace and activated at $800^{\circ} \mathrm{C}$ for $1 \mathrm{~h}$ at a heating rate of $10{ }^{\circ} \mathrm{C} / \mathrm{mn}$ under $\mathrm{N}_{2}$ protection with a flow rate of $96 \mathrm{ml} / \mathrm{min}$. After cooling down to room temperature, the $\mathrm{KOH}$ activated carbon were washed with $0.1 \mathrm{M}$ $\mathrm{HCl}$ at $60^{\circ} \mathrm{C}$ for $1 \mathrm{~h}$ in a shaker to remove the residual $\mathrm{KOH}$ and impurities. Finally, the carbon was washed several times with deionized water until the $\mathrm{pH}$ value became neutral. The carbon material was then dried at $105^{\circ} \mathrm{C}$ overnight in an conveintal oven. The $\mathrm{KOH}$ activated biochar based carbons were denoted as BCK1, BCK2 and BCK3 for biochar/KOH mass ratio of 1:1, 1:2 and 1:3, respectively. For comparison purpose, samples prepared using the same procedure but without $\mathrm{KOH}$ treatment was denoted as BCK0.

\subsection{Preparation of Biochar Based Carbon Nanofibers}

Polyacrylonitrile (PAN, Mw = 150,000, product number: 227056) and $N, N$ dimethylformamide (DMF) (product number: 227056) were purchased from SigmaAldrich. The chemicals were used as received without further purification.

A $10 \mathrm{wt} \%$ bicomponent solution was prepared by dissolving PAN and biochar in the mass ratio of 30:70 in DMF. Biochar was first dispersed in DMF and sonicated for 30 mins, and then added to PAN solution under constant stirring. A $30 \mathrm{ml}$ syringe was next filled with the spinning solution and fitted with a blunt end 15-gauge stainless steel needle. As shown in Fig.1, the electrospinning setup comprised of a high voltage power supply (Series FX, Glassman High Voltage Inc., New Jersey, USA) and a flat movable stainless steel collector plate. The collector setup was fabricated in house and made up of two slides, a computer with a software that controls the motion, and a 30 inch by 30 -inch stainless steel plate. The solution was electrospun at a flow rate of $1 \mathrm{ml} / \mathrm{h}$ and $15 \mathrm{kV}$ and collected on a grounded aluminum foil that was placed at $20 \mathrm{~cm}$ from the tip of the syringe. The resulting electrospun fiber mats were detached from the collector and dried at room temperature in a fume hood for at least $24 \mathrm{~h}$ before further use. The collected electrospun fiber mats were then cut and stacked between $6 \times 6$ in. graphite plates (graphite store), and placed in a furnace (Carbolite HTF 18/8, Sheffield, UK) for further heat treatment. All the samples were stabilized in air from room temperature to $280^{\circ} \mathrm{C}$ at a heating rate of $1{ }^{\circ} \mathrm{C} / \mathrm{min}$ and the temperature was held at $280^{\circ} \mathrm{C}$ for $6 \mathrm{~h}$ to allow complete stabilization. After the stabilized fibers were cooled down to room temperature, they are carbonized in nitrogen at $1200^{\circ} \mathrm{C}$ with a heating rate of $5^{\circ} \mathrm{C} / \mathrm{min}$ and held for $1 \mathrm{~h}$ before cooled down to room temperature.

\subsection{Material Characterization}

The physical characterization of biochar based activated carbon and biochar based ECNFS was conducted by using elemental analysis, nitrogen sorption method, and scanning electron microscopy (SEM). The surface morphology and structure of biochar based carbon and biochar based ECNFs were examined by a Carl Zeiss Auriga-BU FIB field emission scanning electron microscope (FESEM, Oberkochen, Germany). Specific surface area, pore volume, and pore size distribution were measured with a surface area and porosity analyzer (Micromeritics ASAP2020, Georgia, USA). The multilayer 
adsorption model developed by Brunauer-Emmett-Teller (BET) was used to evaluate the surface area (SBET), while the pore size distribution of the sample was evaluated using the Barrett-Joyner-Halenda (BJH) model. Elemental analyses for carbon, hydrogen, and nitrogen contents were determined by using a Perkin-Elmer $2400 \mathrm{CHN} / \mathrm{S}$ analyzer (Waltham, MA, USA).

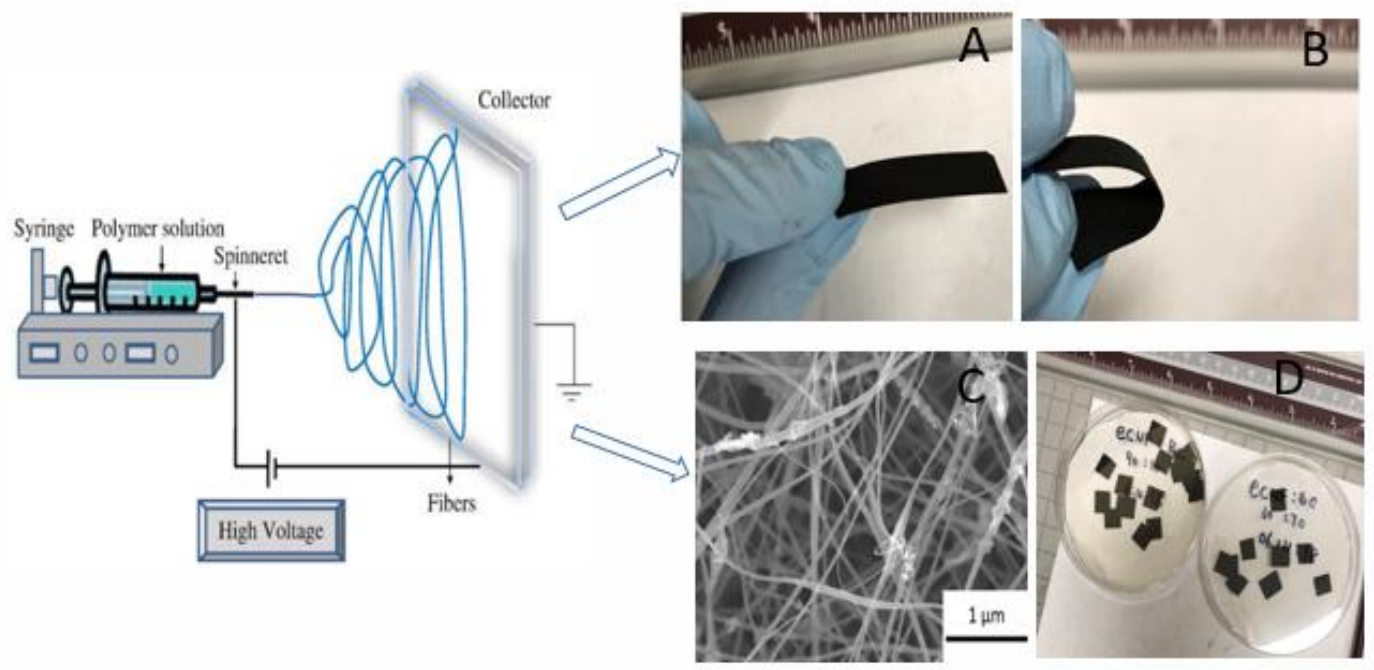

Fig. 1. Schematic illustration for obtaining porous ECNF electrodes using electrospinning method. A) and B) Photo of the ECNF; C) SEM of the ECNF; D) Photo of the electrode

\subsection{Electrochemical Test}

The system for measuring the electrochemical performance of the supercapacitor electrode material is composed of three key components: the electrochemical cell, the electrochemical workstation, and a computer for acquiring test data and control of the workstation. All required electrochemical parameters of the supercapacitor electrode material can be measured using the CHI660E electrochemical workstation $(\mathrm{CH}$ Instruments, Inc., Texas, USA). The three-electrode configuration was assembled using different PAN/Biochar ECNFs mats as working electrodes $\left(\right.$ size $\left.\sim 1.0 \mathrm{~cm}^{2}\right)$. The mass of each carbon fiber mat was measured with an electronic balance. A Pt wire and the $\mathrm{Ag} / \mathrm{AgCl}$ electrode were applied as the counter and reference electrodes, respectively. The working and reference electrodes were first immersed in aqueous electrolyte solution $6 \mathrm{M} \mathrm{NaOH}$ for $24 \mathrm{~h}$; subsequently, cyclic voltammetry (CV) measurement was performed at the scan rate of $5-100 \mathrm{mV} \cdot \mathrm{s}^{-1}$, and the potential window was selected from -0.4 to $0.4 \mathrm{~V}$. For the galvanostatic charge/discharge measurements, the potential window was set from -0.4 to $0.4 \mathrm{~V}$ with current densities from 2 to $0.4 \mathrm{~A} / \mathrm{g}$. The electoral conductivity of the ECNFs was also measured through electrochemical impedance spectroscope (EIS) (i.e., frequency response analysis yields over the frequency ranging from $0.1 \mathrm{~Hz}$ to $100,1000 \mathrm{~Hz}$ with potential amplitude of $5 \mathrm{mV}$ ). All of the electrochemical tests were performed on CHI660E electrochemical testing station.

The specific capacitance of the prepared carbons was calculated from the galvanostatic charge/discharge test at different current density, by the following formula:

$$
c=\frac{2 I \times \Delta t}{m \times \Delta V}
$$


Where, I is the charge or discharge current density, $\Delta t$ is the discharge time, $m$ is the mass of the electrode, and $\Delta V$ is potential voltage, while $C$ is the gravimetric capacitance $(\mathrm{F} / \mathrm{g})$.

\section{Results and Discussion}

\subsection{The Effect of Activation on the Elemental Composition of Biochar}

Table 1 shows the elemental analysis results of the raw biochar and activated biochar carbons obtained from different $\mathrm{KOH}$ ratios. It was observed that the hydrogen, oxygen, nitrogen and sulfur content were all decreased while the carbon content increased after the chemical activation. This indicates an effective carbonization due to the heat treatment during the activation process. The activated biochar BCK2 has the highest carbon content of $78.48 \%$, while BCK3 sample has the highest nitrogen content among the three samples.

Table 1. Elemental analysis results of the biochar and activated biochar carbon

\begin{tabular}{lccccc}
\hline \multicolumn{1}{c}{ Sample } & $\mathbf{C}(\%)$ & $\mathbf{H}(\%)$ & $\mathbf{O}(\%)$ & $\mathbf{N}(\%)$ & $\mathbf{S}(\%)$ \\
\hline Original Biochar (BCK0) & 72.38 & 4.07 & 23.78 & 2.91 & 0.83 \\
Activated Biochar BCK1 (Char: KOH=1:1) & 77.21 & 1.33 & 22.41 & 0.15 & 0.23 \\
Activated Biochar BCK2 (Char:KOH=1:2) & 78.48 & 2.02 & 20.69 & 0.46 & 0.37 \\
Activated Biochar BCK3 (Char:KOH=1:3) & 77.85 & 1.64 & 21.11 & 0.72 & 0.32 \\
\hline
\end{tabular}

\subsection{The Effect of Aactivation on the Pore Characteristics of Biochar}

The morphology and microstructure of the typical samples were analyzed by using SEM. Fig. 2 depicts the surface morphologies of the raw biochar and activated biochars obtained via $\mathrm{KOH}$ treatment with different $\mathrm{KOH}$ ratios. SEM images of BCK1 are not presented here due to the similarity to $\mathrm{BCK} 2$ and $\mathrm{BCK} 3$. It was observed that the raw biochar revealed relatively smooth irregular surfaces. On contrast, the activated biochar showed rougher surfaces with hierarchical and porous structure clearly appeared, indicating more porous structure than the relatively less porous of raw biochar. This change is believed to be originated from $\mathrm{KOH}$ activation under pyrolysis.

In the $\mathrm{KOH}$ activation process, synergistic effects, including etching, gasification, and intercalation were found to be responsible for the pore network formation, porosity enhancement and lattice expansion, respectively [21] . These synergistic effects produced hierarchical porous carbon materials with large SSA and high porosity. Large SSA can increase the double layer capacitance since it provides more chances contacting between the electrode materials and electrolyte. This is desired for the electrode application and were expected to lead to an improvement in capacitance performance. 

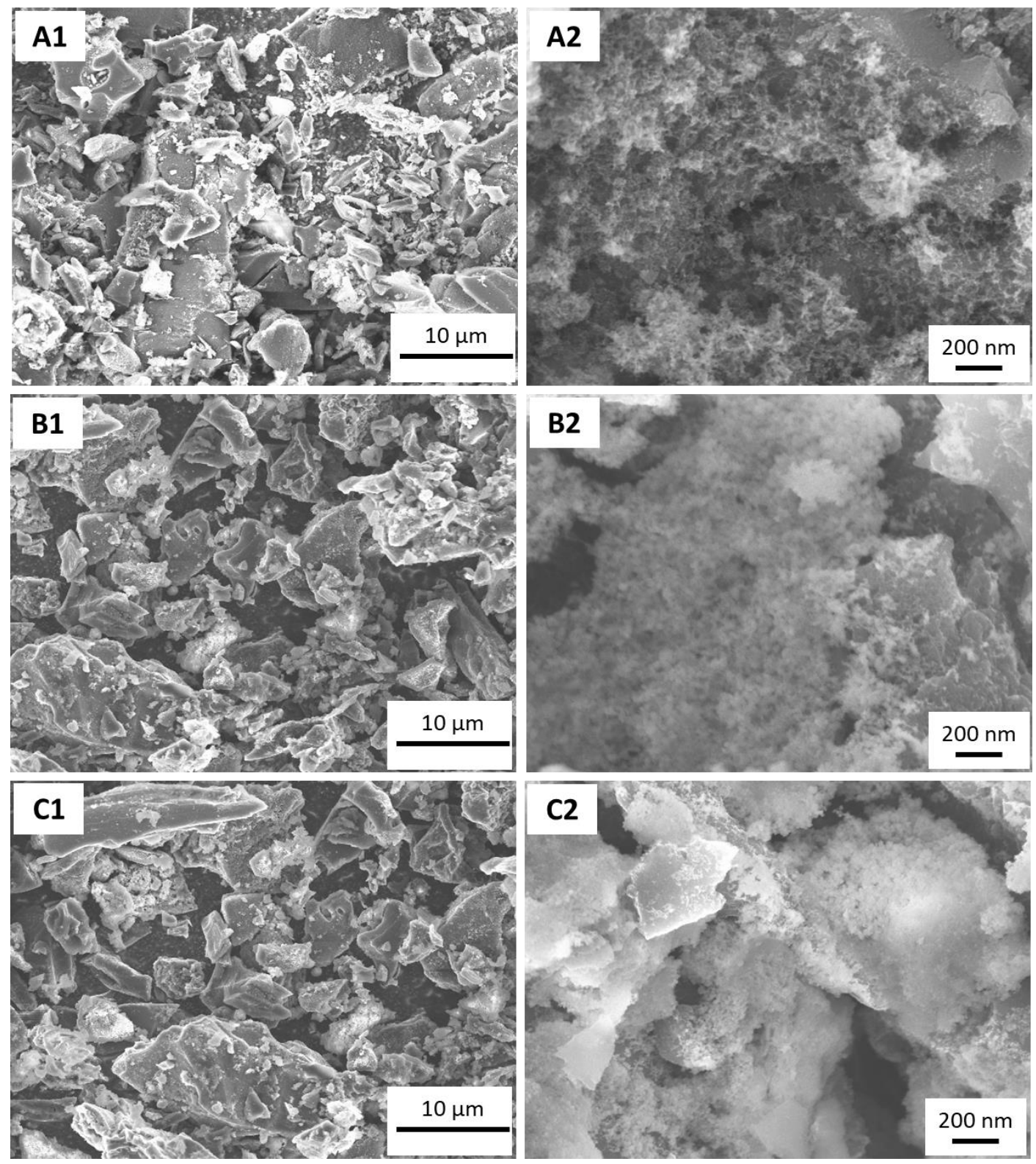

Fig. 2. SEM showing the morphology of biochar $A 1$ and $A 2)$ raw biochar $B C K 0$; $B 1$ and $B 2$ ) BCK2; $\mathrm{C} 1$ and $\mathrm{C} 2) \mathrm{BCK} 3$

To verify the speculation on high SSA and pore structure observed in SEM images, Specific surface area, pore volume, and pore size distribution were measured. Details of the pore characteristic of biochar and activated biochar carbon samples are given in Table 2 . This result indicates that the $\mathrm{KOH}$ activation greatly enhanced the pore development during the activation process. The ratio of $\mathrm{KOH} / \mathrm{bioch}$ ar significantly affects the microstructure of the resultant carbon. The higher the $\mathrm{KOH}$ to biochar ratio, the higher surface area and larger pore volume. The optimized biochar based activated carbon material possesses typical hierarchical porosity composed of multi-leveled pores with high surface area and pore volume up to $2,258 \mathrm{~m}^{2} / \mathrm{g}$ and $1.19 \mathrm{~cm}^{3} / \mathrm{g}$, respectively. 
Table 2 The specific surface area (SSA), volume, pore size, and average diameter of biochar based Carbon

\begin{tabular}{lccc}
\hline \multicolumn{1}{c}{ Sample } & $\begin{array}{c}\text { BET SSA } \\
\left(\mathbf{m}^{2} \mathbf{g}^{-1}\right)\end{array}$ & $\begin{array}{c}\text { Average Pore } \\
\text { Size }(\mathbf{n m})\end{array}$ & $\begin{array}{c}\text { Pore Volume } \\
\left(\mathbf{c m}^{3} \mathbf{g}^{-1}\right)\end{array}$ \\
\hline Original Biochar (BCK0) & 627 & 5.78 & 0.42 \\
Activated Biochar BCK1 (Char: KOH=1:1) & 1083 & 2.4 & 0.65 \\
Activated Biochar BCK2 (Char:KOH=1:2) & 1799 & 2.1 & 0.95 \\
Activated Biochar BCK3 (Char:KOH=1:3) & 2258 & 2.1 & 1.19 \\
\hline
\end{tabular}

\subsection{Morphological and structural properties of Biochar/PAN derived ECNFs.}

An aqueous mixture containing biochar alone could not be electrospun into nanofibers due to the lack of macromolecular structure/entanglements. Uniform PAN solution, by contrast, could be electrospun into PAN nanofibers with an average diameter of $\sim 300 \mathrm{~nm}$ according to our earlier research [12]. As can be seen from Fig. $1 \mathrm{~A} \& \mathrm{~B}$, the resultant PAN/Biochar derived ECNFs is not only free standing but also displays outstanding mechanical flexibility. There are no identifiable cracks or broken fibers can be observed under repeatedly bending, even to $180^{\circ}$. In comparison, the PAN only nanofibers are more fragile. The improved flexibility of biochar derived ECNF might due to the flexible nature of biochar derived from lignocellulosic biomass and high graphitization degree caused by the thermal treatment. The mechanical flexibility would make the PAN/Biochar derived ECNF film suitable for various potential applications such as wearable and portable devices and flexible electronics.

Fig. 3 shows the morphological structures of the PAN/Biochar derived ECNFs. As shown in Fig.3, these PAN/Biochar derived ECNFs consisted of non-woven, randomly oriented carbon nanofibers with a coarse morphology and a large quantity of biochar particles entrapped among the fibers considering the size of most of the biochar particles was large to be incorporated in the fibers. The magnified SEM images in Fig. 3 A2, B2 and C2 further demonstrate that there is certain amount of fine biochar incorporated into the electrospun carbon fibers. The average diameter of the biochar/PAN derived ECNFs was between 56 100 nm, while the average diameter of the PAN alone ECNF was around $300 \mathrm{~nm}$. This significant deduction in fiber diameter might be due to that most of the hydrogen, oxygen and sulfur in the biochar were removed during the thermal treatments, leading to deduction in diameter and the formation of carbonaceous structures. This was also confirmed by the elemental analysis result listed in Table 1. SEM results also revealed that with the increase of $\mathrm{KOH}$ ratio in the chemical activation of the biochar particles, the average diameters of the ECNFs gradually increased from $\sim 56 \mathrm{~nm}$ to $\sim 100 \mathrm{~nm}$. This could be because after activation, the biochar particles are easier to be broken down into smaller sizes during sonication. Hence a lot more are embedded in the fibers thereby increasing their average sizes. 

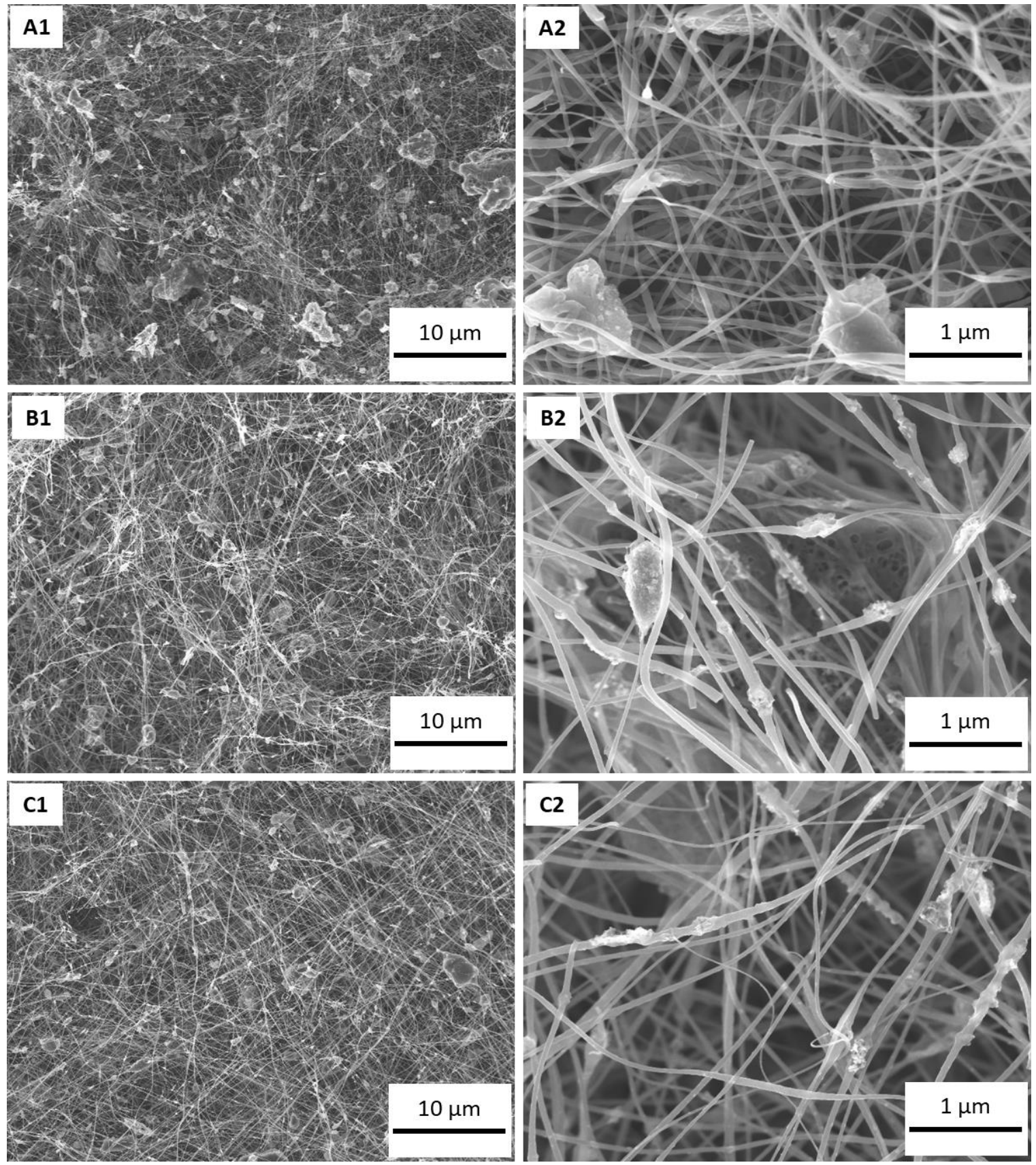

Fig. 3. SEM of electrospun carbon nanofibers (ECNFs) from electrospinning PAN and biochar bicomponent solutions: $\mathrm{A} 1$ and $\mathrm{A} 2$ ) ECNF from PAN and raw biochar BCK0; B1 and B2) ECNF from PAN and BCK2; $\mathrm{C} 1$ and $\mathrm{C} 2$ ) ECNF from PAN and BCK3

The porous structure and size distribution of PAN/Biochar derived ECNFs were analyzed by BET measurement. It was evident that incorporation of biochar particles into electrospun PAN nanofibers led to larger BET SSA and larger pore volume than the PAN alone ECNFs (Table 3). The larger SSA allowed an efficient contact of nanofibers with electrolyte, which is beneficial for electronic and ionic transport. As shown in Table 3, the PAN derived ECNFs has the lowest specific surface areas of $11 \mathrm{~m}^{2} / \mathrm{g}$, while the 
PAN/Biochar derived ECNFs exhibited much higher SSA than the PAN derived ECNFs, with the highest SSA of $505 \mathrm{~m}^{2} / \mathrm{g}$ from the PAN/BCK2 ECNFs. It's believed that the high porosity of the activated carbon is attributed to the significant change of the SSA and pore volume of the resultant ECNFs.

Table 3 The specific surface area (SSA), volume, pore size and average diameter of PAN/Biochar Derived ECNFs

\begin{tabular}{lcccc}
\hline Sample & $\begin{array}{c}\text { BET SSA } \\
\left(\mathbf{m}^{\mathbf{2}} \mathbf{g}^{-\mathbf{1}}\right)\end{array}$ & $\begin{array}{c}\text { Average Pore Size } \\
(\mathbf{n m})\end{array}$ & $\begin{array}{c}\text { Pore Volume } \\
\left(\mathbf{c m}^{\mathbf{3}} \mathbf{g}^{-\mathbf{1}}\right)\end{array}$ & $\begin{array}{c}\text { Average Fiber } \\
\text { Diameter }(\mathbf{n m})\end{array}$ \\
\hline PAN ECNF & 11 & 8.6 & 0.024 & 300 \\
\hline PAN/BCK0 ECNF & 97.37 & 7.23 & 0.18 & $56.36 \pm 6.93$ \\
PAN/BCK2 ECNF & 505.92 & 2.49 & 0.31 & $95.17 \pm 18.61$ \\
PAN/BCK3 ECNF & 381.11 & 2.77 & 0.26 & $99.71 \pm 16.35$ \\
\hline
\end{tabular}

\subsection{Electrochemical performance}

The electrochemical capacitive performance of the PAN/Biochar derived ECNFs were evaluated by cyclic voltammetry and galvanostatic charge/discharge measurement. Typical CV curves of the ECNFs electrode were collected at a scan rate between 5$100 \mathrm{mV} / \mathrm{s}$ within a potential window from -0.4 to $0.4 \mathrm{~V}$ in $6 \mathrm{M} \mathrm{KOH}$ aqueous electrolyte. Fig. 4 (a) shows the cyclic voltammetry loops of PAN derived and different PAN/Biochar derived ECNFs at scan rate of $100 \mathrm{mv} / \mathrm{s}$. At a scan rate of $100 \mathrm{mV} / \mathrm{s}, \mathrm{CV}$ curve of the PAN derived ECNF exhibited a long and narrow non-rectangular shape and enclosed a very small loop area, suggesting very small electrochemical double layer capacitive behavior. This result can be attributed to the low conductivity and hydrophobicity of the PAN derived ECNF [16]. CV curves of PAN/Biochar derived ECNFs exhibited Quasirectangular shape and enclosed much larger loop area, indicating these biochar incorporated ECNFs have higher gravimetric capacitance and would be more desired electrode materials for the supercapacitor. Among the four ECNFs, the PAN/BCK13 ECNF has the CV curve with the largest loop area, suggesting the highest gravimetric capacitance. The sample of ECNF PAN/BCK3 was further scanned at varied scan rate of $5-100 \mathrm{mV} / \mathrm{s}$ (Fig. 4b). With the increase of voltage sweep rate, the CV curves well maintain the quasi-rectangular shape with little distortion, indicating a reversible supercapacitor behavior in organic electrolyte within the potential range. This can be attributed to the excellent conductivity and low mass-transport resistance of the samples.

The charge and discharge curves of different ECNF electrodes were obtained at constant current density of $0.5 \mathrm{~A} / \mathrm{g}$ with potential window from -0.4 to $0.4 \mathrm{~V}$ in $6 \mathrm{M} \mathrm{KOH}$ aqueous electrolyte (Fig. 4c). The charge/discharge curves of these ECNFs were approximately isosceles, indicating excellent capacitive performance such as great electrochemical stability and reversibility [22]. The galvanostatic charge/discharge behavior of PAN/BCK3 ECNFs was further investigated at various current densities of 1 $\mathrm{A} / \mathrm{g}$ and $2 \mathrm{~A} / \mathrm{g}$ (Fig. 4d). The charge and discharge curves were almost linear and the isosceles triangles indicated excellent supercapacitor behaviors. 

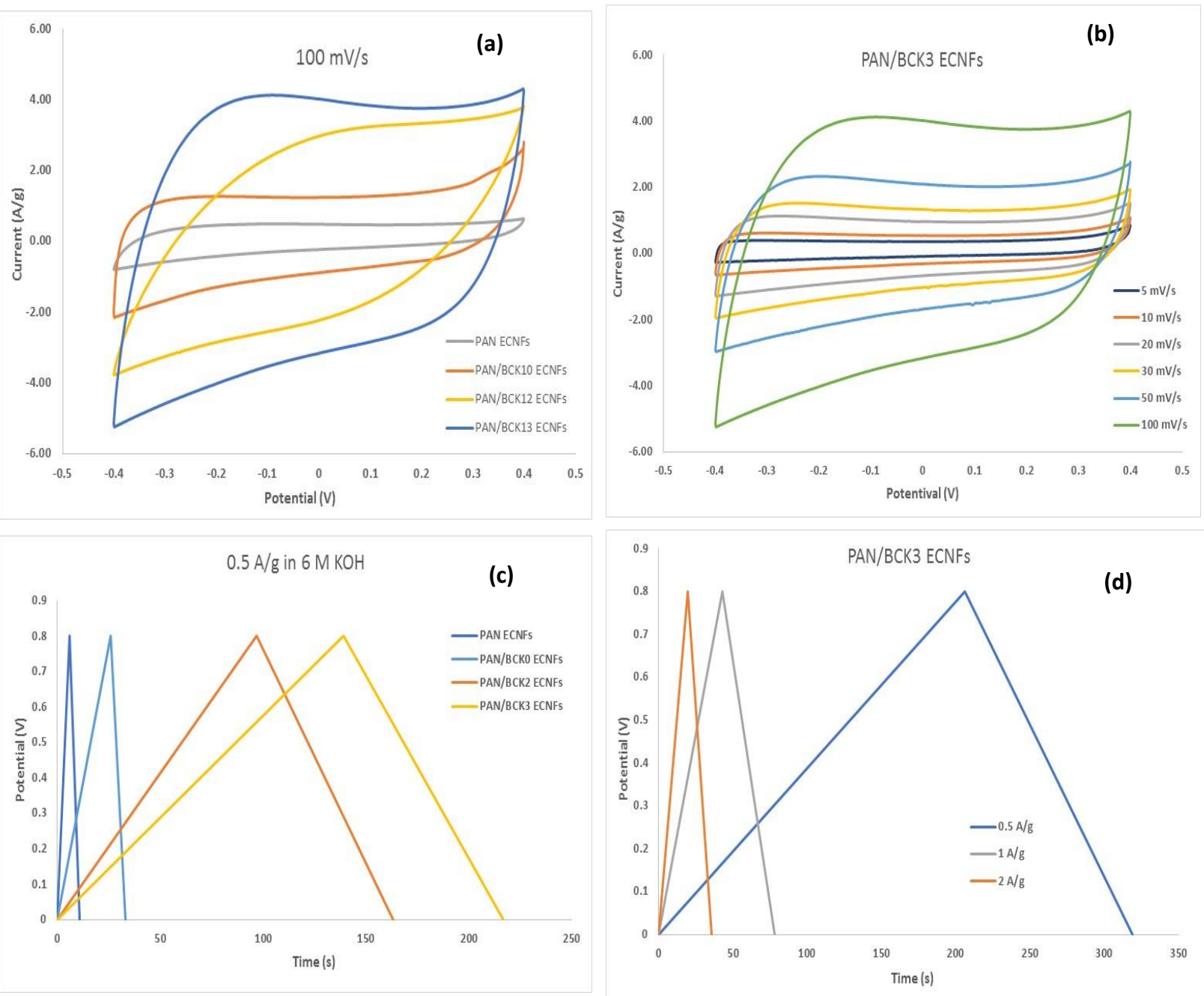

Fig. 4. Electrochemical characterization of different Biochar-based ECNF electrodes in $6 \mathrm{M} \mathrm{KOH}$ aqueous electrolyte: (a) Cyclic voltammetry loops at scan rate of $100 \mathrm{mv} / \mathrm{s}$; (b) Cyclic voltammetry loops of PAN/BCK3 ECNFs at different voltage sweeping rates; (c) Galvanostatic charge/discharge curves at the current density of $0.5 \mathrm{~A} / \mathrm{g}$; and (d) Galvanostatic charge/discharge curves of PAN/BCK3 ECNFs at different current densities.

The gravimetric capacitance of PAN/BCK0, PAN/BCK2, PAN/BCK3 ECNFs electrodes at the current density of $0.5 \mathrm{~A} / \mathrm{g}$ were 32,87 , and $108 \mathrm{~F} / \mathrm{g}$, respectively. With the increase of $\mathrm{KOH}$ ratio in the chemical activation of biochar, the decrease in average pore size and increase in of the SSA can be contributed to the increase of the gravimetric capacitance. The gravimetric capacitances of PAN/BCK3 ECNFs at $0.5,1$, and $2 \mathrm{~A} / \mathrm{g}$ were 108, 88, and $80 \mathrm{~F} / \mathrm{g}$ respectively. As the current density increased, the corresponding capacitance reduced slightly. It was observed that $\sim 78 \%$ of the initial capacitance was retained at a relatively large current density of $2 \mathrm{~A} / \mathrm{g}$ when PAN/BCK3 ECNFs was used as the electrode. It is worth noting that the capacitance of PAN/BCK3 ECNFs is substantially higher than the most recent reported values for using freestanding ENCFs as electrode materials for supercapacitor, such as ECNF/ $\mathrm{MnO}_{2}(83.3 \mathrm{~F} / \mathrm{g})$ [23], HTL Biochar/PAN ECNFs (37.6 F/g), and lignin-derived ECNFs (64 F/g) [15]. This 
suggests that the ECNFs made from activated biochar might be innovative carbon nanomaterials for energy storage applications.

Long term cycling performance is a crucial parameter for electrode material of supercapacitor. The stability of capacitance performance of PAN/BCK3 ECNFs was investigated through galvanostatic/charge/discharge test for 1,000 cycles within a potential window from $-0.4 \mathrm{~V}$ to $0.4 \mathrm{~V}$ at high current density of $1 \mathrm{~A} / \mathrm{g}$ (Fig. 5a). The gravimetric capacitance was merely reduced by $4 \%$ after 1,000 cycles of charge and discharge, indicating that the PAN/BCK3 ECNFs is electrochemical stable and durable.
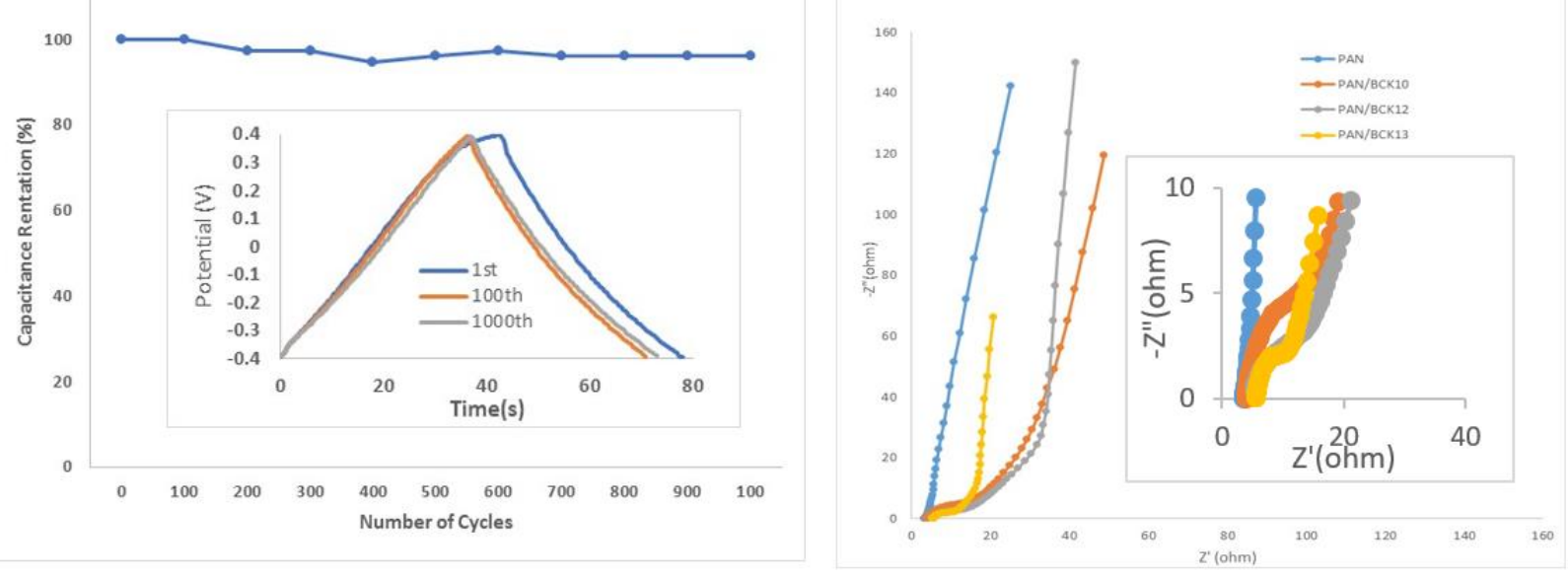

Fig. 5. a) Cycling stability of PAN/BCK3 ECNFs electrode material under current density of $1 \mathrm{~A} / \mathrm{g}$ with embedded charge/discharge curves of $1^{\text {st }}, 100^{\text {th }}$, and $1000^{\text {th }}$ cycles; b) Nyquist plots of different biochar based ECNF electrodes

To further investigate the electrochemical behavior of ECNFs electrodes, electrochemical impedance spectroscopy (EIS) was carried out as shown in Fig. 5(b). EIS is a powerful technique to obtain the information on both the characteristics of pores and the dynamic ion diffusion in porous electrodes. For biochar based ECNFs, the impedance spectra included a distorted semicircle in the high frequency regime and a liner part nearly vertical to the realistic impedance axis at the low frequency regime. No evident semicircle corresponding to charge transfer resistance was found for PAN alone ECNFs. In the high frequency range, the plot intersection at real part $Z$ ' is associated with the electrolyte resistance $\left(R_{S}\right)$ and the square resistance of the samples. The semicircle region in the plot curve corresponds to the charge transfer resistance $\left(R_{c t}\right)$, which represents the migration rate of ions at the interface between the solution and the electrode surface.

The diameters of the semicircles are listed in the following order: PAN/BCK0>PAN/BCK1>PAN/BCK3. The smaller diameter of semicircle in the EIS spectrum of PAN/BCK3 ECNFs indicates lower charge transfer resistance. In the low frequency region, the line has finite slope representing the diffusive resistivity of the electrolyte within the pore of the electrode, as represented by Warburg impedance. The slope of PAN/BCK3 ECNFs approached to an ideally straight line implying outstanding capacitive performance thanks to the enhanced accessibility of the ions [16]. All these results revealed that the PAN/BCK3 ECNFs have the lowest internal resistance and the 
best ion transition and diffusion among all three bio-char based ECNFs, in good agreement with the $\mathrm{CV}$ curves and specific galvanostatic plots.

\section{CONCLUSIONS}

Highly flexible porous biochar based ECNFs were fabricated by electrospinning technique and explored as electrode material. It was found that $\mathrm{KOH}$ plays an important role on the creation of porous structures. The increase of $\mathrm{KOH}$ loading amount in the activation process would lead to the increase of specific surface area of the activated char as well as the ECNFs after fabrication. The biochar based ECNFs not only showed a large SSA, but also exhibited high flexibility and an improved conductivity. As designed, the biochar based ECNFs showed high specific capacitance, high cycle stability and excellent electrochemical performance. This research illustrates that this biochar based ECNF is a promising electrode material for high performance supercapacitor.

\section{ACKNOWLEDGMENTS}

The authors thank USDA-CSREES-Evans-Allen Project (NCX-303-5-17-130-1) for the financial support.

\section{CONFLICTS OF INTEREST}

The authors declare that there is no conflict of interests regarding the publication of this paper.

\section{REFERENCES}

[1] Wang, K., Xu, M., Gu, Z., Ahrenkiel, P., Lee, J., Gibbons, W., Croat, J., and Fan, Q. (2016). Pyrrole modified biomass derived hierarchical porous carbon as high performance symmetrical supercapacitor electrodes. International Journal of Hydrogen Energy, 41(30), 13109-13115. DOI: 10.1016/j.ijhydene.2016.05.090

[2] Jin, H., Wang, X., Shen, Y., and Gu, Z. (2014). A high-performance carbon derived from corn stover via microwave and slow pyrolysis for supercapacitors. Journal of Analytical and Applied Pyrolysis, 110, 18-23. DOI:

10.1016/j.jaap.2014.07.010

[3] Dyatkin, B., Presser, V., Heon, M., Lukatskaya, M. R., Beidaghi, M., and Gogotsi, Y. (2013). Development of a Green Supercapacitor Composed Entirely of Environmentally Friendly Materials. ChemSusChem, 6(12), 2269-2280. DOI: $10.1002 /$ cssc. 201300852

[4] Qiu, Z., Wang, Y., Bi, X., Zhou, T., Zhou, J., Zhao, J., Miao, Z., Yi, W., Fu, P., and Zhuo, S. (2018). Biochar-based carbons with hierarchical micro-meso-macro porosity for high rate and long cycle life supercapacitors. Journal of Power Sources, 376, 82-90. DOI: 10.1016/j.jpowsour.2017.11.077 
[5] Liu, M.-C., Kong, L.-B., Zhang, P., Luo, Y.-C., and Kang, L. (2012). Porous wood carbon monolith for high-performance supercapacitors. Electrochimica Acta, 60, 443-448. DOI: 10.1016/j.electacta.2011.11.100

[6] Cheng, B.-H., Zeng, R. J., and Jiang, H. (2017). Recent developments of postmodification of biochar for electrochemical energy storage. Bioresource Technology, 246, 224-233. DOI: 10.1016/j.biortech.2017.07.060

[7] Gao, F., Shao, G., Qu, J., Lv, S., Li, Y., and Wu, M. (2015). Tailoring of porous and nitrogen-rich carbons derived from hydrochar for high-performance supercapacitor electrodes. Electrochimica Acta, 155, 201-208. DOI: 10.1016/j.electacta.2014.12.069

[8] Zhang, L. L., and Zhao, X. S. (2009). Carbon-based materials as supercapacitor electrodes. Chemical Society Reviews, 38(9), 2520-2531. DOI: 10.1039/B813846J

[9] Xiu, S., Shahbazi, A., and Li, R. (2017). Characterization, Modification and Application of Biochar for Energy Storage and Catalysis: A Review. Trends in Renewable Energy, 3(1), 86-101. DOI: 10.17737/tre.2017.3.1.0033

[10] Zhang, S., Su, Y., Zhu, S., Zhang, H., and Zhang, Q. (2018). Effects of pretreatment and $\mathrm{FeCl} 3$ preload of rice husk on synthesis of magnetic carbon composites by pyrolysis for supercapacitor application. Journal of Analytical and Applied Pyrolysis, 135, 22-31. DOI: 10.1016/j.jaap.2018.09.026

[11] Li, W., Chen, D., Li, Z., Shi, Y., Wan, Y., Wang, G., Jiang, Z., and Zhao, D. (2007). Nitrogen-containing carbon spheres with very large uniform mesopores: The superior electrode materials for EDLC in organic electrolyte. Carbon, 45(9), 1757-1763. DOI: 10.1016/j.carbon.2007.05.004

[12] Aboagye, A., Liu, Y., Ryan, J. G., Wei, J., and Zhang, L. (2018). Hierarchical carbon composite nanofibrous electrode material for high-performance aqueous supercapacitors. Materials Chemistry and Physics, 214, 557-563. DOI: 10.1016/j.matchemphys.2018.05.009

[13] Duan, P., and Savage, P. E. (2011). Hydrothermal Liquefaction of a Microalga with Heterogeneous Catalysts. Industrial \& Engineering Chemistry Research, 50(1), 52-61. DOI: 10.1021/ie100758s

[14] Du, X., Zhao, W., Wang, Y., Wang, C., Chen, M., Qi, T., Hua, C., and Ma, M. (2013). Preparation of activated carbon hollow fibers from ramie at low temperature for electric double-layer capacitor applications. Bioresource Technology, 149, 31-37. DOI: 10.1016/j.biortech.2013.09.026

[15] Lai, C., Zhou, Z., Zhang, L., Wang, X., Zhou, Q., Zhao, Y., Wang, Y., Wu, X.-F., Zhu, Z., and Fong, H. (2014). Free-standing and mechanically flexible mats consisting of electrospun carbon nanofibers made from a natural product of alkali lignin as binder-free electrodes for high-performance supercapacitors. Journal of Power Sources, 247, 134-141. DOI: 10.1016/j.jpowsour.2013.08.082

[16] Liu, Y., Zhou, J., Chen, L., Zhang, P., Fu, W., Zhao, H., Ma, Y., Pan, X., Zhang, Z., Han, W., and Xie, E. (2015). Highly Flexible Freestanding Porous Carbon Nanofibers for Electrodes Materials of High-Performance All-Carbon Supercapacitors. ACS Applied Materials \& Interfaces, 7(42), 23515-23520. DOI: 10.1021/acsami.5b06107

[17] Sevilla, M., Yu, L., Zhao, L., Ania, C., and Titiricic, M.-M. (2014). Surface Modification of CNTs with N-Doped Carbon: An Effective Way of Enhancing Their Performance in Supercapacitors. ACS Sustainable Chem. Eng., 2 (4), 1049 1055. DOI: $10.1021 / \mathrm{sc} 500069 \mathrm{~h}$ 
[18] Cheng, Y., Lu, S., Zhang, H., Varanasi, C. V., and Liu, J. (2012). Synergistic Effects from Graphene and Carbon Nanotubes Enable Flexible and Robust Electrodes for High-Performance Supercapacitors. Nano Letters, 12(8), 42064211. DOI: $10.1021 / \mathrm{nl} 301804 \mathrm{c}$

[19] Hao, J., Zhong, Y., Liao, Y., Shu, D., Kang, Z., Zou, X., He, C., and Guo, S. (2015). Face-to-face self-assembly graphene/MnO2 nanocomposites for supercapacitor applications using electrochemically exfoliated graphene. Electrochimica Acta, 167, 412-420. DOI: 10.1016/j.electacta.2015.03.098

[20] Li, R., Shahbazi, A., Wang, L., Zhang, B., Chung, C.-C., Dayton, D., and Yan, Q. (2018). Nanostructured molybdenum carbide on biochar for $\mathrm{CO} 2$ reforming of CH4. Fuel, 225, 403-410. DOI: 10.1016/j.fuel.2018.03.179

[21] Wang, J., and Kaskel, S. (2012). KOH activation of carbon-based materials for energy storage. Journal of Materials Chemistry, 22(45), 23710-23725. DOI: 10.1039/C2JM34066F

[22] Razaq, A., Nyholm, L., Sjödin, M., Strømme, M., and Mihranyan, A. (2012). Paper-based energy-storage devices comprising carbon fiber-reinforced polypyrrole-cladophora nanocellulose Composite electrodes. Advanced Energy Materials, 2(4), 445-454. DOI: 10.1002/aenm.201100713

[23] Ma, X., Kolla, P., Zhao, Y., Smirnova, A. L., and Fong, H. (2016). Electrospun lignin-derived carbon nanofiber mats surface-decorated with $\mathrm{MnO} 2$ nanowhiskers as binder-free supercapacitor electrodes with high performance. Journal of Power Sources, 325, 541-548. DOI: 10.1016/j.jpowsour.2016.06.073

Article copyright: (C) 2019 Shuangning Xiu, Spero Gbewonyob, Abolghasem Shahbazi1, and Lifeng Zhang. This is an open access article distributed under the terms of the Creative Commons Attribution 4.0 International License, which permits unrestricted use and distribution provided the original author and source are credited. 